\title{
Genetic interaction between profilin and myosin II reveals a potential role for myosin II in actin filament disassembly in vivo
}

Paola Zambon ${ }^{1}$, Saravanan Palani ${ }^{1}$, Shekhar Sanjay Jadhav ${ }^{2}$, Pananghat Gayathri ${ }^{2}$, and Mohan K. Balasubramanian ${ }^{1}$

${ }^{1}$ Division of Biomedical Sciences, Warwick Medical School, University of Warwick, Coventry CV4 7AL, UK

${ }^{2}$ Biology Division, Indian Institute of Science Education and Research (IISER), Pune, Maharashtra 411008, India

Author for Correspondence: Mohan K. Balasubramanian (m.k.balasubramanian@warwick.ac.uk) 


\section{Abstract:}

The actin cytoskeleton plays a variety of roles in eukaryotic cell physiology, ranging from cell polarity and migration to cytokinesis. Key to the function of the actin cytoskeleton is the mechanisms that control its assembly, stability, and turnover. Through genetic analyses in fission yeast, we found that, myo2-S1 (myo2-G515D), a myosin II mutant allele was capable of rescuing lethality caused by compromise of mechanisms involved in actin cable / ring assembly and stability. The mutation in myo2-S1 affects the activation loop of Myosin II, which is involved in physical interaction with subdomain 1 of actin and in stimulating the ATPase activity of Myosin. Consistently, actomyosin rings in myo2-S1 cell ghosts were severely compromised in contraction upon ATP addition, suggesting that Myo2-S1p was defective in actin binding and / or motor activity. These studies strongly suggest a role for Myo2p in actin cytoskeletal disassembly and turnover, and that compromise of this activity leads to genetic suppression of mutants defective in actin cable assembly / stability.

\section{Introduction:}

Actin is a highly conserved cytoskeletal polymer forming protein that is key to a vast array of physiological processes in the three domains of life. In eukaryotes, the actin cytoskeleton plays essential roles in cell polarity, morphogenesis, migration, and cytokinesis (Pollard and Wu 2010, Cheffings, Burroughs et al. 2016, Misu, Takebayashi et al. 2017, Rottner, Faix et al. 2017, Skruber, Read et al. 2018). A balance of factors that control filament nucleation and stability and those that promote its disassembly exquisitely regulates the functions of the actin cytoskeleton (Lee and Dominguez 2010). Over the past three decades, the fission yeast Schizosaccharomyces pombe has emerged as an attractive organism for the study of the actin cytoskeleton and its role in cell polarity and division (Pollard and Wu 2010, Cheffings, Burroughs et al. 2016, Chiou, Balasubramanian et al. 2017). This is particularly due to the fact that many of the actin cytoskeletal proteins can be characterized through easily identifiable lethal mutant phenotypes (Wertman, Drubin et al. 1992, Holtzman, Wertman et al. 1994, Balasubramanian, McCollum et al. 1998).

In fission yeast, the Arp2/3 complex nucleates actin patches (Pelham and Chang 2001, Sirotkin, Berro et al. 2010), whereas linear actin cables and cytokinetic actomyosin rings are assembled by formins For3 (actin cables) (Feierbach and Chang 2001) and Cdc12p (cytokinetic actomyosin rings) (Chang, Drubin et al. 1997) and the actin-binding protein Cdc3-profilin (Balasubramanian, Hirani et al. 1994). The coiled-coil actin binding protein Cdc8 tropomyosin ensures stability of actin cables and actomyosin rings (Liu and Bretscher 1989, Balasubramanian, Helfman et al. 1992, Gunning, Hardeman et al. 2015). The fission yeast actin cytoskeleton undergoes dramatic disassembly and turnover (Kovar, Sirotkin et al. 2011). Treatment of cells with the actin polymerization inhibitor latrunculin A causes complete loss of actin cables, actomyosin rings, and actin patches (in that order) (Pelham and Chang 2001, Pelham and Chang 2002). While the actin severing protein Adf1 (cofilin) plays a key role in actin cytoskeletal disassembly (Nakano and 
Mabuchi 2006, Pavlov, Muhlrad et al. 2007), the fact that the actin cables and rings still turnover, albeit slowly, in adf1-1 mutants (Nakano and Mabuchi 2006) suggests that other mechanisms should also exist to disassemble the actin cytoskeleton.

Myo2-S1 (Myo2 G515D) was isolated in a genetic screen for suppressors of the high temperature lethality of cdc3-124 that led to characterization of the Arp2/3 complex protein Sop2 (Balasubramanian, Feoktistova et al. 1996, Wong, Naqvi et al. 2000), although Myo2-S1 mutant has not been previously characterized. Here we characterize the cellular and molecular basis of the suppression of cdc3-124 and other conditions that cause partial loss of the actin cytoskeleton. These studies point to an in vivo role for myosin II in actin filament disassembly.

\section{Results and Discussion:}

\section{myo2-S1 suppresses the ts lethality of $c d c 3-124$}

Previous work has shown that cdc3-124 is defective in actomyosin ring assembly and colony formation above $32^{\circ} \mathrm{C}$ (Balasubramanian, Feoktistova et al. 1996). To investigate and recreate the suppression of cdc3-124 by myo2-S1, we freshly generated cdc3-124 myo2-S1 from a cross between a myo2-S1 parent (obtained after three rounds of back crossing with wild-type cells) and cdc3-124. The newly created cdc3-124 myo2-S1 strain was compared to wild-type and to cdc3124 and myo2-S1 single mutants. As expected, wild-type cells formed colonies at all temperatures tested, whereas cdc3-124 failed to form colonies at and above $32^{\circ} \mathrm{C}$ (Figure $1 \mathrm{~A}$ ). myo2-S1 was able to form colonies at all temperatures tested, although these cells displayed cytokinesis defects at all temperatures tested (described in later sections) (Figure 1A). Importantly, the recreated cdc3124 myo2-S1 was able to form colonies at $34^{\circ} \mathrm{C}$ and did so poorly, even at the higher restrictive temperature of $36^{\circ} \mathrm{C}$ (Figure $1 \mathrm{~A}$ ). The genetic screen (Balasubramanian, Feoktistova et al. 1996) also identified a different allele of myo2, myo2-S2 (Myo2 E679K)(Wong, Naqvi et al. 2000), which rescued cdc3-124 (Figure 1A). We recreated the cdc3-124 myo2-S2, using a back-crossed myo2$\mathrm{S} 2$ parent, and found that it rescued cdc3-124 at $34^{\circ} \mathrm{C}$, but barely rescued it at $36^{\circ} \mathrm{C}$ (Figure $1 \mathrm{~A}$ ). The well-characterized myo2-E1 allele (Balasubramanian, McCollum et al. 1998) did not rescue cdc3-124 either at $34^{\circ} \mathrm{C}$ or $36^{\circ} \mathrm{C}$. Since myo2-S1 better suppressed the cdc3-124 defect, we characterized this mutant further in the rest of this study.

The strains described above were grown in liquid medium and stained with calcofluor white to visualize the division septa. Whereas normal (or nearly normal) division septa, that appeared as a disc in tilted images were detected at $24^{\circ} \mathrm{C}$ in all strains, complete septa that appeared as a disc in tilted images were found in wild-type, myo2-S1, and cdc3-124 myo2-S1, but not in cdc3-124, at $34^{\circ} \mathrm{C}$ (Figure 1B). Instead, patches of septum material that did not create a barrier between the two daughters were observed in $c d c 3-124$ at $34^{\circ} \mathrm{C}$ (Figure 1B). This experiment established that 
compromise of myo2, through the myo2-S1 mutation, led to full rescue of the septation defect of cdc3-124.

Cdc3-profilin (Balasubramanian, Hirani et al. 1994), in conjunction with Cdc12-formin (Chang, Drubin et al. 1997) plays a key role linear actin filament nucleation during cytokinesis (Kovar, Kuhn et al. 2003), whereas Cdc8-tropomyosin (Balasubramanian, Helfman et al. 1992) plays a role in actin cable and ring stabilization (Gunning, Hardeman et al. 2015, Khaitlina 2015). We therefore tested if the observed suppression was specific to Cdc3-profilin defects or if myo2-S1 also suppressed defects in $c d c 12$ and $c d c 8$. To test this, we generated double mutants cdc8-110 myo2$\mathrm{S} 1$ and $c d c 12-112$ myo2-S1. We found that $c d c 8-110$ was suppressed at $34^{\circ} \mathrm{C}$ and tiny "pin-prick" colonies were observed even at $36^{\circ} \mathrm{C}$ (Figure 1C). cdc12-112 was not suppressed at either $34^{\circ} \mathrm{C}$ or at $36^{\circ} \mathrm{C}$ (Figure $\left.1 \mathrm{C}\right)$.

Next, we stained wild-type, cdc3-124, myo2-S1 and cdc3-124 myo2-S1 with CF633-phalloidin and DAPI to observe the actin cytoskeleton and nuclei (Figure 1D and E). This analysis showed that whereas actin rings were not observed in cdc3-124, actin rings were observed in mitotic wild-type, myo2-S1, and cdc3-124 myo2-S1 mutants (Figure 1D). The rings in myo2-S1 were more diffuse compared to those in wild-type consistent with a role for Myo2p in actomyosin ring assembly. These experiments established that compromise of Myo2p function led to suppression of the defective actomyosin ring assembly, septum assembly, and colony formation in cdc3-124 (Figure $1 \mathrm{E})$. The partial suppression of $c d c 8-110$ by myo2-S1 (Figure 1C), suggested that myo2-S1 suppression is not specific to cdc3-124 and that it also suppress defects in other actin assembly / stability factors.

To investigate the mechanism of suppression of cdc3-124 by myo2-S1, as well as to characterize the phenotypic consequences of myo2-S1, if any, we generated wild-type, cdc3-124, myo2-S1, and cdc3-124 myo2-S1, expressing Rlc1-3GFP (actomyosin ring marker) and mCherry-Atb2 (mitotic spindle marker). At the permissive temperature of $24^{\circ} \mathrm{C}$, actomyosin ring assembly and contraction were indistinguishable in wild-type and cdc3-124 cells, whereas both processes were slightly slower in myo2-S1 and cdc3-124 myo2-S1 cells (Figure S1A and B). Ring assembly in wild-type at $34^{\circ} \mathrm{C}$ took $17 \pm 6.8$ minutes and ring contraction took $18.7 \pm 2.1$ minutes (Figure 2B and $\mathrm{C}$ ). As expected, actomyosin rings did not assemble in cdc3-124 cells although Rlc1-3GFP accumulated first in nodes and then in multiple spots and bar-like structures (Figure $2 \mathrm{~A}$ ). The myo2-S1 mutant showed a range of phenotypes pertaining to cytokinesis. First, in $\sim 50 \%$ of the cells, ring assembly was delayed and took $\sim 1.8$ times longer (30.2 \pm 4 minutes) than in wild-type cells and fully compacted rings were observed only in cells with elongated mitotic spindles (Figure $2 \mathrm{~A}$ class I and class III and Figure $2 \mathrm{~B}$ ). Second, the remaining $\sim 50 \%$ of the cells made abnormal actomyosin structures that did not compact into a ring structure (Figure $2 \mathrm{~A}$ class IV). Ring 
contraction was uniformly slower in cells that appeared to have normal rings ( $38.3 \pm 6.9$ minutes) (Figure 2C). Furthermore, ring contraction was asymmetric in nearly two-thirds of cells that appeared to have a normal looking ring (Figure 2A class III). The cdc3-124 myo2-S1 double mutant largely resembled myo2-S1 single mutant and rings of normal appearance were detected in $\sim 42 \%$ of the cells and these rings took $26.2 \pm 1.9$ minutes to assemble, while the rest of the cells assembled Rlc-3GFP bundles that did not compact into a ring (Figure 2A and B). Ring contraction in cdc3-124 myo2-S1 cells was almost always asymmetric and the process took almost three times the time taken in wild-type cells $(56.3 \pm 26.8$ minutes) (Figure $2 \mathrm{~A}$ and $\mathrm{C}$ ). These experiments established that myo2-S1 was compromised for both known physiological roles of Myo2p, i.e. actomyosin ring assembly and contraction, and that the compromise of one of its known activities contributed to the genetic suppression of cytokinesis and colony formation defects of cdc3-124.

\section{ATP-dependent contraction is dramatically slowed in actomyosin rings isolated from myo2- S1 cells}

The mutated myosin allele, Myo2-S1, possesses a point mutation resulting in the replacement of Gly515 with an aspartate in the L50 subdomain of Myo2p motor domain. G515D is located at the beginning of HR helix just at the end of activation loop (Varkuti, Yang et al. 2012). This glycine might play a role in the conformational flexibility of the activation loop, thereby contributing to the loop's orientation that facilitates actin binding. In order to understand the effect of the mutation, we analyzed the position equivalent to G515 in various myosin structures. In the cryoEM structure of myosin bound to actin filaments (5JLH), G515 was observed to be at the beginning of the activation loop that interacted with $\mathrm{N}$-terminal end of an actin monomer (Figure 3A). Modeling of the G515D mutation on myosin motor domain structure (1VOM) showed a salt bridge formation between the mutated Asp515 and Arg509 residues (data not shown). Arg520 in C. elegans muscle myosin, corresponding to Lys510 in S. pombe Myo2p, has been shown to be crucial for binding with the N-terminal region of actin (Varkuti, Yang et al. 2012). Furthermore, Arg520 mutation affected the ATPase activity of myosin motor domain indicating that Arg520 interaction with actin is essential for motor domain function (Varkuti, Yang et al. 2012). In a sequence alignment of 237 non-redundant myosin sequences, glycine is highly conserved and a positively charged residue (at least one arginine or lysine) is a conserved feature of the activation loop (Figure 3B). Unavailability or misorientation of the positively charged side chain (Lys or Arg) to interact with actin in the G515D mutant (Myo2-S1) might weaken its interaction with actin, leading to abnormalities in ring assembly. The mutation might also affect the motor activity in the defective rings due to the absence of ATPase stimulation upon actin interaction, thus affecting the actomyosin ring contraction rates.

Given these considerations based on structural analysis of myo2-S1, we wanted to test the biochemical properties of Myo2-S1p, especially as it pertains with actin binding or motor activity. 
We were unable to purify this protein in sufficient quantities for biochemical studies (data not shown). As an alternative, we therefore used the permeabilized spheroplast assay, in which actomyosin rings contract in an ATP and myosin II dependent manner (Mishra, Kashiwazaki et al. 2013, Huang, Mishra et al. 2016). We prepared cell ghosts from wild-type, myo2-S1 and cdc3-124 myo2-S1 cells expressing Rlc1-3GFP that were grown at $24^{\circ} \mathrm{C}$. We carried out two sets of experiments, one at $24^{\circ} \mathrm{C}$ (Figure S2A) and the other at $34^{\circ} \mathrm{C}$ (Figure $4 \mathrm{~A}$ and S2B). Actomyosin rings in wild-type cell ghosts were stably maintained and contracted rapidly upon ATP addition at $24^{\circ} \mathrm{C}$ (Figure S2A and $4 \mathrm{C}$ ). However, actomyosin rings became extremely unstable upon introduction of the myo2-S1 mutation, suggesting that Myo2-S1p may interact weakly with actin causing the ring to be unstable in the context of a cell ghost (Figure S2A). We therefore stabilized actomyosin rings by incubation of cell ghosts with the actin-stabilizing compound Jasplakinolide (Jas) (Holzinger 2009). We next treated Jas-stabilized actomyosin rings in cell ghosts with ATP. As observed in the absence of Jas, rings in wild-type ghosts contracted rapidly at $34^{\circ} \mathrm{C}$ (Figure $4 \mathrm{~A}$ and B). However, even though actomyosin rings were stably maintained in myo2-S1 and cdc3-124 myo2-S1, they either failed to contract or were extremely slow to contract (Figure 4A and B). A similar trend was observed when Jas-stabilized wild-type, myo2-S1, and cdc3-124 myo2-S1 ghosts were incubated with ATP even at the lower temperature of $24^{\circ} \mathrm{C}$ (Figure $4 \mathrm{C}$ ). This is similar to our previous observations with cell ghosts of the well characterized temperature-sensitive myo2E1 allele, rings from which fail to contract upon ATP addition, even at the permissive temperature of $24^{\circ} \mathrm{C}$ (Mishra, Kashiwazaki et al. 2013, Palani, Chew et al. 2017). In addition, we found that Jasstabilized actomyosin rings in cell ghosts prepared from cdc3-124 myo2-S2 (which suppresses cdc3-124, but less well compared to myo2-S1) also underwent very slow ATP-dependent contraction at $34^{\circ} \mathrm{C}$ (Figure S2B and C) and at $24^{\circ} \mathrm{C}$ (Figure S2D). These observations established that loss of myosin II motor activity strongly correlated with the suppression of actomyosin ring assembly / stability defect in cdc3-124. The fact that two different mutants, which show defects in ATP-dependent ring contraction in vitro, suppress cdc3-124 suggests that reduced actin binding and / or motor activity rather than some other defect in Myo2p contributes to the rescue of cdc3124.

\section{myo2-S1 suppresses direct pharmacological perturbation of the actin cytoskeleton with}

\section{Latrunculin A}

Previous work has shown that myosin II can break actin filaments in vitro both by stretching or buckling (Murrell and Gardel 2012, Vogel, Petrasek et al. 2013) due to its motor activity. We have shown that myosin II mutants defective in ATP dependent contraction (and by inference in motor activity) suppress defects caused by partial loss of proteins contributing actin cable and ring assembly / stability. We reasoned that shorter or unstable actin filaments may be protected for longer periods in the cdc3-124 myo2-S1, cdc8-110 myo2-S1, and cdc3-124 myo2-S2 mutants, 
which in turn may contribute to the observed suppression of the cytokinesis and colony formation defects. We therefore tested if direct pharmacological perturbation of actin cables and actomyosin rings (which are composed of formin generated linear actin filaments) by treatment with low doses of Latrunculin A (Ayscough, Stryker et al. 1997) can be reversed by compromise of Myo2p. Previously, we have shown that treatment of wild-type cells with low doses of Latrunculin A ( 0.25 $\mu \mathrm{M})$ leads to cytokinesis defects (Mishra, Karagiannis et al. 2004). We plated serial dilutions of wild-type, cdc3-124, myo2-S1, and cdc3-124 myo2-S1 with a series of doses of Latrunculin A and incubated these at $30^{\circ} \mathrm{C}$ and $34^{\circ} \mathrm{C}$ (Figure $5 \mathrm{~A}$ ). We found that wild-type cells were capable of robust colony formation up to $0.25 \mu \mathrm{M}$ Latrunculin $\mathrm{A}$, but did not form colonies at $0.375 \mu \mathrm{M}$ Latrunculin A (Figure 5A). Consistent with a role for Cdc3p profilin in actin cable and actomyosin ring assembly, cdc3-124 mutants were hypersensitive to $0.25 \mu \mathrm{M}$ Latrunculin A. Interestingly, myo2-S1 mutants were able to form robust colonies at all temperatures tested up to $0.375 \mu \mathrm{M}$ Latrunculin A (Figure 5A), establishing that compromise of Myo2p function reverses the deleterious effects caused by actin perturbation. The cdc3-124 myo2-S1 was also capable of colony formation at $30^{\circ} \mathrm{C}$ at $0.375 \mu \mathrm{M}$ Latrunculin $\mathrm{A}$, although at $34^{\circ} \mathrm{C}$, this strain only grew on plates containing $0.125 \mu \mathrm{M}$ Latrunculin A (Figure 5A). The inability of the cdc3-124 to form colonies at and above $0.25 \mu \mathrm{M}$ Latrunculin A potentially reflects an enhanced actin cytoskeletal defect due to the additive effect of loss of Cdc3p and Latrunculin A. CF633-Phalloidin staining of liquid cultures of the four strains described above showed that actin cables, rings, and patches were present at 0 time point in all four strains (Figure 5B and C). After a 3 hours incubation in $0.375 \mu \mathrm{M}$ Latrunculin A, actin patches were observed in all 4 strains. However, actin cables / rings were not detected in wild-type or cdc3-124 but were clearly detected in myo2-S1 and cdc3-124 myo2-S1 cells (Figure 5B and C). Collectively, these experiments established that partial genetic or pharmacological perturbation of actin cables and rings can be suppressed by motor-activity defective Myo2p.

In summary, in this study we provide evidence for a role for myosin II in actin filament disassembly through the use of genetic, structural, and in vitro analyses. Previous work has shown that single actin filaments and networks of actin filaments are broken and disassembled by myosin II through buckling (Murrell and Gardel 2012, Vogel, Petrasek et al. 2013). Previous work in S. japonicus has shown that isolated cytokinetic rings break into a series of clusters in a myosin II dependent manner, which is reversed and normal contraction upon ATP addition ensues, when the isolated cytokinetic rings are pre-incubated with Jasplakinolide (Chew, Huang et al. 2017). Our work may provide in vivo evidence for a role for myosin II in actin filament disassembly and turnover, also consistent with the work in cytokinetic mammalian cells (Murthy and Wadsworth 2005). It is possible that formin-generated actin filaments are short and unstable in profilin and tropomyosin mutants and upon exposure of cells with low doses of Latrunculin A. In this scenario, compromise of myosin II, which normally destabilizes actin filaments, rescues the instability of actin filaments and, in turn, the cytokinesis defects in cdc3-124, cdc8-110 and Latrunculin A treated cells. 


\section{Materials and methods}

\section{Yeast genetics and culture methods}

Cells were grown and cultured at $24^{\circ} \mathrm{C}$ in yeast extract medium (YES) as described previously (Moreno, Klar et al. 1991). The presence of designated mutations on each used strain has been verified by PCR and DNA sequencing.

\section{PFA fixation and fluorescence microscopy}

S. pombe cells, growing at $24^{\circ} \mathrm{C}$ in YES medium, were either fixed in mid-log phases or shifted to $34^{\circ} \mathrm{C}$ for 3-4 hours before fixation. Cells were fixed in a $4 \%$ paraformaldehyde solution for 12 minutes at room temperature and, after two washes with 1X PBS, permeabilized with $1 \%$ Triton X100 for 10 minutes. Cells were washed twice with 1 X PBS and stained with either DAPI (4',6diamidino-2-phenylindole, from Life Technologies), for the visualization of the nucleus, or CF633Phalloidin (CF633, from Biotium Inc) to visualize actin structures. To visualize the septa, fixed $S$. pombe cells were directly incubated with Calcofluor white (CW, from Sigma-Aldrich). Still images were acquired using a spinning disk confocal microscope (specifications described below in livecell imaging section) and processed using imaging software Fiji.

\section{Live-cell imaging}

Mid-log phase cells were grown at $24^{\circ} \mathrm{C}$ in YES medium and, when necessary, shifted at $34^{\circ} \mathrm{C}$ for 3-4 hours before imaging acquisition of live cells in a temperature-controlled incubation chamber. Time-lapse images were acquired for 3-4 hours using a spinning disk confocal microscope (Andor Revolution XD imaging system, equipped with a 100x oil immersion 1.45NA Nikon Plan Apo lambda, and a confocal unit Yokogawa CSU-X1, EMCCD detector (Andor iXON) and Andor iQ acquisition software). Cells were imaged in a CellASIC microfluidic yeast plates (Y04C and D size), where 15 Z-stacks of $0.5 \mu \mathrm{m}$ thickness images were acquired at 1-minute intervals for Rlc1-3GFP (myosin regulatory light chain, used as actomyosin ring marker) and Atb2-mCherry (alpha tubulin 2, used as cell cycle marker). PRISM 6.0 software (GraphPad) was used for quantification and the statistical significance was determined using Student's t-test $\left.{ }^{* * * *} \mathrm{P}<0.0001\right)$.

\section{Isolation of actomyosin rings and ATP-dependent contraction}

Actomyosin rings were isolated as described previously (Mishra, Kashiwazaki et al. 2013, Huang, Mishra et al. 2016). Time-lapse imaging of Rlc1-3GFP were acquired as described before in a CellASIC microfluidic yeast plates (Y04D), where 21 Z-stacks of $0.5 \mu \mathrm{m}$ thickness images were acquired while treating the cells with $0.5 \mathrm{mM} \mathrm{ATP}$, either at $24^{\circ} \mathrm{C}$ or after shifting 10 minutes the isolated ring at $34^{\circ} \mathrm{C}$. Fiji imaging software was used to process the acquired images, obtaining the maximum intensity projection of the Z-stacks. 


\section{Drug treatments in cells and isolated actomyosin rings}

YES agar plates in Figure 5A were prepared by the addition of different concentrations of latrunculin A (Lat-A; Enzo Life Sciences), where cells were successively being spotted at 10 -fold serial dilutions. For the treatment in Figure 5B, cells were grown in liquid YES medium at $24^{\circ} \mathrm{C}$ and successively shifted at $34^{\circ} \mathrm{C}$, in the presence of $0.375 \mu \mathrm{M}$ Lat- $\mathrm{A}$ in the medium, for 3 hours before fixation.

Isolated actomyosin rings were treated, when indicated, with jasplakinolide (jasp; Enzo Life Sciences) by the addition of the drug to the isolated rings at the final concentration of $20 \mu \mathrm{M}$.

\section{Table of strains used in this study}

\begin{tabular}{|l|l|}
\hline MBY17 & cdc3-124 leu1-32 ura4-D18 h+ \\
\hline MBY54 & myo2-S2 ade6-21 ura4-D18 h- \\
\hline MBY53 & my02-S1 ade6-21 ura4-D18 leu1-32 h- \\
\hline MBY109 & cdc8-110 ade6-210 ura4-D18 leu1-32 h- \\
\hline MBY144 & cdc12-112 ura4-D18 leu1-32 h- \\
\hline KGY247 & ade6-210 ura4-D18 leu1-32 h+ \\
\hline MBY12728 & myo2-S1 cdc3-124 ade6-21 ura4-D18 leu1-32 \\
\hline MBY12730 & $\begin{array}{l}\text { myo2-S1 rlc1-3GFP ::kanMx6 mCherry-atb2::hph ade6-21 ura4-D18 leu1- } \\
\text { 32 h+ }\end{array}$ \\
\hline MBY12732 & $\begin{array}{l}\text { myo2-S1 cdc3-124 rlc1-3GFP::kanMx6 mCherry-atb2::hph ade6-21 ura4- } \\
\text { D18 leu1-32 h- }\end{array}$ \\
\hline MBY12734 & myo2-S2 cdc3-124 ade6-21 ura4-D18 leu1-32 \\
\hline MBY12736 & $\begin{array}{l}\text { myo2-S2 rlc1-3GFP::kanMx6 mCherry-atb2::hph ade6-21 ura4-D18 leu1- } \\
32\end{array}$ \\
\hline MBY12738 & $\begin{array}{l}\text { myo2-S2 cdc3-124 rlc1-3GFP::kanMx6 mCherry-atb2::hph ade6-21 ura4- } \\
\text { D18 leu1-32 h+ }\end{array}$ \\
\hline MBY12928 & myo2-S1 cdc8-110 ade6-21 ura4-D18 leu1-32 \\
\hline MBY12930 & myo2-S1 cdc12-112 ade6-21 ura4-D18 leu1-32 \\
\hline MBY12931 & myo2-S2 cdc8-110 ade6-21 ura4-D18 leu1-32 \\
\hline MBY12933 & myo2-S2 cdc12-112 ade6-21 ura4-D18 leu1-32 \\
\hline
\end{tabular}

\section{Acknowledgements}

This work was funded by research grants from Wellcome Trust (WT101885MA) and the European Research Council (GA 671083 - ACTOMYOSIN RING) to MKB. PG was supported by research 
grant from SERB Women Excellence Award and computation facilities supported by the Collaborative Research Grant from CEFIPRA (Indo-French Centre for Promotion of Advanced Research). SJ acknowledges INSPIRE for fellowship. We thank Dr. Bernardo Chapa-y-Lazo for help with image analysis.

\section{References}

Ayscough, K. R., J. Stryker, N. Pokala, M. Sanders, P. Crews and D. G. Drubin (1997). "High rates of actin filament turnover in budding yeast and roles for actin in establishment and maintenance of cell polarity revealed using the actin inhibitor latrunculin-A." J Cell Biol 137(2): 399-416.

Balasubramanian, M. K., A. Feoktistova, D. McCollum and K. L. Gould (1996). "Fission yeast Sop2p: a novel and evolutionarily conserved protein that interacts with Arp3p and modulates profilin function." EMBO J 15(23): 6426-6437.

Balasubramanian, M. K., D. M. Helfman and S. M. Hemmingsen (1992). "A new tropomyosin essential for cytokinesis in the fission yeast S. pombe." Nature 360(6399): 84-87.

Balasubramanian, M. K., B. R. Hirani, J. D. Burke and K. L. Gould (1994). "The Schizosaccharomyces pombe cdc3+ gene encodes a profilin essential for cytokinesis." $\mathrm{J}$ Cell Biol 125(6): 1289-1301.

Balasubramanian, M. K., D. McCollum, L. Chang, K. C. Wong, N. I. Naqvi, X. He, S. Sazer and K. L. Gould (1998). "Isolation and characterization of new fission yeast cytokinesis mutants." Genetics 149(3): 1265-1275.

Chang, F., D. Drubin and P. Nurse (1997). "cdc12p, a protein required for cytokinesis in fission yeast, is a component of the cell division ring and interacts with profilin." J Cell Biol 137(1): 169182.

Cheffings, T. H., N. J. Burroughs and M. K. Balasubramanian (2016). "Actomyosin Ring Formation and Tension Generation in Eukaryotic Cytokinesis." Curr Biol 26(15): R719-R737.

Chew, T. G., J. Huang, S. Palani, R. Sommese, A. Kamnev, T. Hatano, Y. Gu, S. Oliferenko, S. Sivaramakrishnan and M. K. Balasubramanian (2017). "Actin turnover maintains actin filament homeostasis during cytokinetic ring contraction." J Cell Biol 216(9): 2657-2667.

Chiou, J. G., M. K. Balasubramanian and D. J. Lew (2017). "Cell Polarity in Yeast." Annu Rev Cell Dev Biol 33: 77-101.

Crooks, G. E., G. Hon, J. M. Chandonia and S. E. Brenner (2004). "WebLogo: a sequence logo generator." Genome Res 14(6): 1188-1190.

Feierbach, B. and F. Chang (2001). "Roles of the fission yeast formin for3p in cell polarity, actin cable formation and symmetric cell division." Curr Biol 11(21): 1656-1665.

Gunning, P. W., E. C. Hardeman, P. Lappalainen and D. P. Mulvihill (2015). "Tropomyosin master regulator of actin filament function in the cytoskeleton." J Cell Sci 128(16): 2965-2974.

Holtzman, D. A., K. F. Wertman and D. G. Drubin (1994). "Mapping actin surfaces required for functional interactions in vivo." J Cell Biol 126(2): 423-432. 
Holzinger, A. (2009). "Jasplakinolide: an actin-specific reagent that promotes actin polymerization." Methods Mol Biol 586: 71-87.

Huang, J., M. Mishra, S. Palani, T. G. Chew and M. K. Balasubramanian (2016). "Isolation of Cytokinetic Actomyosin Rings from Saccharomyces cerevisiae and Schizosaccharomyces pombe." Methods Mol Biol 1369: 125-136.

Khaitlina, S. Y. (2015). "Tropomyosin as a Regulator of Actin Dynamics." Int Rev Cell Mol Biol 318: 255-291.

Kovar, D. R., J. R. Kuhn, A. L. Tichy and T. D. Pollard (2003). "The fission yeast cytokinesis formin Cdc12p is a barbed end actin filament capping protein gated by profilin." J Cell Biol 161(5): 875887.

Kovar, D. R., V. Sirotkin and M. Lord (2011). "Three's company: the fission yeast actin cytoskeleton." Trends Cell Biol 21(3): 177-187.

Lee, S. H. and R. Dominguez (2010). "Regulation of actin cytoskeleton dynamics in cells." Mol Cells 29(4): 311-325.

Liu, H. P. and A. Bretscher (1989). "Disruption of the single tropomyosin gene in yeast results in the disappearance of actin cables from the cytoskeleton." Cell 57(2): 233-242.

Mishra, M., J. Karagiannis, S. Trautmann, H. Wang, D. McCollum and M. K. Balasubramanian (2004). "The Clp1p/Flp1p phosphatase ensures completion of cytokinesis in response to minor perturbation of the cell division machinery in Schizosaccharomyces pombe." J Cell Sci 117(Pt 17): 3897-3910.

Mishra, M., J. Kashiwazaki, T. Takagi, R. Srinivasan, Y. Huang, M. K. Balasubramanian and I. Mabuchi (2013). "In vitro contraction of cytokinetic ring depends on myosin II but not on actin dynamics." Nat Cell Biol 15(7): 853-859.

Misu, S., M. Takebayashi and K. Miyamoto (2017). "Nuclear Actin in Development and Transcriptional Reprogramming." Front Genet 8: 27.

Moreno, S., A. Klar and P. Nurse (1991). "Molecular genetic analysis of fission yeast Schizosaccharomyces pombe." Methods Enzymol 194: 795-823.

Murrell, M. P. and M. L. Gardel (2012). "F-actin buckling coordinates contractility and severing in a biomimetic actomyosin cortex." Proc Natl Acad Sci U S A 109(51): 20820-20825.

Murthy, K. and P. Wadsworth (2005). "Myosin-II-dependent localization and dynamics of F-actin during cytokinesis." Curr Biol 15(8): 724-731.

Nakano, K. and I. Mabuchi (2006). "Actin-depolymerizing protein Adf1 is required for formation and maintenance of the contractile ring during cytokinesis in fission yeast." Mol Biol Cell 17(4): 19331945.

Palani, S., T. G. Chew, S. Ramanujam, A. Kamnev, S. Harne, Y. L. B. Chapa, R. Hogg, M. Sevugan, M. Mishra, P. Gayathri and M. K. Balasubramanian (2017). "Motor Activity Dependent and Independent Functions of Myosin II Contribute to Actomyosin Ring Assembly and Contraction in Schizosaccharomyces pombe." Curr Biol 27(5): 751-757. 
Pavlov, D., A. Muhlrad, J. Cooper, M. Wear and E. Reisler (2007). "Actin filament severing by cofilin." J Mol Biol 365(5): 1350-1358.

Pelham, R. J. and F. Chang (2002). "Actin dynamics in the contractile ring during cytokinesis in fission yeast." Nature 419(6902): 82-86.

Pelham, R. J., Jr. and F. Chang (2001). "Role of actin polymerization and actin cables in actinpatch movement in Schizosaccharomyces pombe." Nat Cell Biol 3(3): 235-244.

Pollard, T. D. and J. Q. Wu (2010). "Understanding cytokinesis: lessons from fission yeast." Nat Rev Mol Cell Biol 11(2): 149-155.

Rottner, K., J. Faix, S. Bogdan, S. Linder and E. Kerkhoff (2017). "Actin assembly mechanisms at a glance." J Cell Sci 130(20): 3427-3435.

Sievers, F. and D. G. Higgins (2018). "Clustal Omega for making accurate alignments of many protein sequences." Protein Sci 27(1): 135-145.

Sievers, F., A. Wilm, D. Dineen, T. J. Gibson, K. Karplus, W. Li, R. Lopez, H. McWilliam, M. Remmert, J. Soding, J. D. Thompson and D. G. Higgins (2011). "Fast, scalable generation of highquality protein multiple sequence alignments using Clustal Omega." Mol Syst Biol 7: 539.

Sirotkin, V., J. Berro, K. Macmillan, L. Zhao and T. D. Pollard (2010). "Quantitative analysis of the mechanism of endocytic actin patch assembly and disassembly in fission yeast." Mol Biol Cell 21(16): 2894-2904.

Skruber, K., T. A. Read and E. A. Vitriol (2018). "Reconsidering an active role for G-actin in cytoskeletal regulation." J Cell Sci 131(1).

Varkuti, B. H., Z. Yang, B. Kintses, P. Erdelyi, I. Bardos-Nagy, A. L. Kovacs, P. Hari, M. Kellermayer, T. Vellai and A. Malnasi-Csizmadia (2012). "A novel actin binding site of myosin required for effective muscle contraction." Nat Struct Mol Biol 19(3): 299-306.

Vogel, S. K., Z. Petrasek, F. Heinemann and P. Schwille (2013). "Myosin motors fragment and compact membrane-bound actin filaments." Elife 2: e00116.

Waterhouse, A. M., J. B. Procter, D. M. Martin, M. Clamp and G. J. Barton (2009). "Jalview Version 2--a multiple sequence alignment editor and analysis workbench." Bioinformatics 25(9): 11891191.

Wertman, K. F., D. G. Drubin and D. Botstein (1992). "Systematic mutational analysis of the yeast ACT1 gene." Genetics 132(2): 337-350.

Wong, K. C., N. I. Naqvi, Y. lino, M. Yamamoto and M. K. Balasubramanian (2000). "Fission yeast Rng3p: an UCS-domain protein that mediates myosin II assembly during cytokinesis." J Cell Sci 113 ( Pt 13): 2421-2432. 


\section{Figure legends}

Figure 1: myo2-S1 suppresses the lethality of $c d c 3-124$ at the non-permissive temperature.

A) 10-fold serial dilutions of wild-type, cdc3-124, myo2-S1, cdc3-124 myo2-S1, myo2-S2 and cdc3-124 myo2-S2 were spotted onto YEA agar plates and grown for 3 days at $24^{\circ} \mathrm{C}, 34^{\circ} \mathrm{C}$ and $36^{\circ} \mathrm{C}$.

B) Calcofluor white staining was used to visualize the septum of wild-type, cdc3-124, myo2-S1 and cdc3-124 myo2-S cells, fixed at $24^{\circ} \mathrm{C}$ or after 4 hours shift at $34^{\circ} \mathrm{C}$. In the first column it is shown the acquired front view image of the septum, while in the second column it's displayed the face-on view of the septum, which was generated with Fiji software (as illustrated by the cartoon). Scale bar represent $3 \mu \mathrm{m}$.

C) 10-fold serial dilutions of wild-type, cdc8-110, cdc12-112, myo2-S1, cdc8-110 myo2-S1 and cdc12-112 myo2-S1 were spotted onto YEA agar plates and grown for 3 days at $24^{\circ} \mathrm{C}$, $34^{\circ} \mathrm{C}$ and $36^{\circ} \mathrm{C}$.

D) Cells were grown at $24^{\circ} \mathrm{C}$ and shifted for 4 hours at $34^{\circ} \mathrm{C}$ before PFA fixation. CF633phalloidin and DAPI were used to visualize actin structures and the nucleus respectively, of wild-type, cdc3-124, myo2-S1and cdc3-124 myo2-S1. Arrows indicated normal actomyosin rings while asterisks indicated abnormal rings. Scale bar represent $5 \mu \mathrm{m}$.

E) Quantification of CF633-phalloidin and DAPI staining in (D) is shown. Cells with 2 nuclei were classified depending on the presence of either no ring (2N/no ring), 1 normal ring (2N/1 ring), 1 abnormal ring (2N/abnormal ring) or multiple nuclei and rings (MN/M rings).

\section{Figure 2: Actomyosin ring assembly and contraction is partially restored in myo2-S1 and} cdc3-124 myo2-S1 at $34^{\circ} \mathrm{C}$.

A) Time-lapse series of wild-type, cdc3-124, myo2-S1and cdc3-124 myo2-S1 cells expressing Rlc1-3GFP as a contractile ring marker and mCherry- atb2 as a cell-cycle stage marker. Cells were grown at $24^{\circ} \mathrm{C}$ and shifted 3 hours at $34^{\circ} \mathrm{C}$ before being imaged at $34^{\circ} \mathrm{C}$. More than 20 cells were imaged and quantified for each strain. On the site of each montage it is indicated the percentage of the different cytokinetic behaviours categorized into class I (normal actomyosin ring - AMR), class II (failed AMR assembly), class III (normal AMR assembly and asymmetrical AMR contraction) and class IV (abnormal AMR structures). Images shown are maximum-intensity projections of Z-stacks. Time indicated in minutes. Scale bars represent $3 \mu \mathrm{m}$.

B) Quantification of the time necessary for actomyosin ring assembly of class I and class III cells imaged in (A). Statistical significance was calculated by Student's test $\left({ }^{* * *} p<0.0001\right)$. Error bars represent SD.

C) Quantification of the time necessary for actomyosin ring contraction of class I and class III cells imaged in (A). Statistical significance was calculated by Student's test $\left({ }^{* * *} \mathrm{p}<0.0001\right)$. Error bars represent SD. 


\section{Figure 3: Structural basis of S. pombe Myo2-S1 (G515D) mutation}

A) Gly515 in S. pombe Myo2 (equivalent to Gly549 of human myosin 14 in the actomyosin complex structure of PDB ID: 5JLH) is positioned at the boundary of the activation loop at the myosin-actin interface. Inset shows the zoomed view of the interaction between the activation loop and the N-terminus of actin protomer. Gly549 and Arg543, corresponding to Gly515 and Lys510 of $S$. pombe Myo2, are shown in stick representation. The figure was generated using PyMol.

B) Conservation information of Gly515 and Lys510 depicted on a sequence logo representation. The figure was generated using WebLogo (Crooks, Hon et al. 2004) using a sequence alignment of 237 non-redundant myosin sequences aligned using ClustalO (Sievers, Wilm et al. 2011, Sievers and Higgins 2018). The sequence corresponding to the activation loop was selected using JalView (Waterhouse, Procter et al. 2009).

\section{Figure 4: Actomyosin rings isolated from myo2-S1 and cdc3-124 myo2-S1 cells do not} undergo ATP-dependent contraction.

A) In vitro isolated actomyosin rings were prepared from wild-type $(n=17)$, myo2-S1 $(n=14)$, and cdc3-124 myo2-S1 $(n=20)$ cells grown at $24^{\circ} \mathrm{C}$ in the presence of $20 \mu \mathrm{M}$ jasplakinolide (jasp). Isolated rings were shifted 15 minutes at $34^{\circ} \mathrm{C}$ before proceeding with imaging. Ring contraction experiments were performed at $34^{\circ} \mathrm{C}$ and contraction was activated by addition of $0.5 \mathrm{mM}$ ATP (indicated by the red bar). Images shown are maximum intensity projections of Z-stacks. Time indicated in minutes. Scale bar represent $5 \mu \mathrm{m}$.

B) Percentage of rings that either contracted normally or presented slow/no contraction of strains imaged at $34^{\circ} \mathrm{C}$ illustrated in $(\mathrm{A})$.

C) Percentage of rings that either contracted normally or presented slow/no contraction of strains imaged at $24^{\circ} \mathrm{C}$ illustrated in $(\mathrm{A})$.

\section{Figure 5: myo2-S1 suppresses perturbations of actin induced by Latrunculin A}

A) 10-fold serial dilutions of wild-type, cdc3-124, myo2-S1and cdc3-124 myo2-S1 cells were spotted onto YEA agar plates containing different concentrations of latrunculin-A (Lat-A) and grown for 3 days at $24^{\circ} \mathrm{C}, 34^{\circ} \mathrm{C}$ and $36^{\circ} \mathrm{C}$.

B) Cells were grown at $24^{\circ} \mathrm{C}$ and shifted for 3 hours at $34^{\circ} \mathrm{C}$ in the presence of $0.375 \mu \mathrm{M}$ of Lat-A before PFA fixation. CF633-phalloidin was used to visualize actin structures of wildtype, cdc3-124, myo2-S1 and cdc3-124 myo2-S1 cells. Arrows indicated the presence of actin cables. Scale bar represent $5 \mu \mathrm{m}$.

C) Quantification of the presence of actin cables or actomyosin ring structure detected with CF633-phalloidin staining in (D). More than 200 cells were imaged for wild-type, cdc3-124, myo2-S1and cdc3-124 myo2-S1. 


\section{Supplemental Figure Legends}

\section{Supplemental 1}

A) Quantification of the time necessary for actomyosin ring assembly of class I and class III cells imaged at $24^{\circ} \mathrm{C}$ in wild-type, cdc3-124, myo2-S1and cdc3-124 myo2-S1 strains. Statistical significance was calculated by Student's test. Error bars represent SD.

B) Quantification of the time necessary for actomyosin ring contraction of class I and class III cells imaged at $24^{\circ} \mathrm{C}$ in wild-type, cdc3-124, myo2-S1and cdc3-124 myo2-S1 strains. Statistical significance was calculated by Student's test. Error bars represent SD.

\section{Supplemental 2}

A) In vitro isolated actomyosin rings were prepared from wild-type $(n=17)$ and myo2-S1 $(n=15)$ cells in the absence of jasplakinolide. Ring contraction experiments were performed at $24^{\circ} \mathrm{C}$ and contraction was activated by addition of $0.5 \mathrm{mM}$ ATP (indicated by the red bar). Images shown are maximum intensity projections of Z-stacks. Time indicated in minutes. Scale bar represent $5 \mu \mathrm{m}$.

B) In vitro isolated actomyosin rings were prepared from cdc3-124 myo2-S2 cells $(\mathrm{n}=23)$ grown at $24^{\circ} \mathrm{C}$ in the presence of $20 \mu \mathrm{M}$ jasp. Isolated rings were shifted 15 minutes at $34^{\circ} \mathrm{C}$ before proceeding with imaging. Ring contraction experiments were performed at $34^{\circ} \mathrm{C}$ and contraction was activated by addition of $0.5 \mathrm{mM}$ ATP (indicated by the red bar). Images shown are maximum intensity projections of Z-stacks. Time indicated in minutes. Scale bar represent $5 \mu \mathrm{m}$.

C) Percentage of rings isolated from wild-type, myo2-S2 and cdc3-124 myo2-S2 cells that either contracted normally or presented slow/no contraction imaged at $34^{\circ} \mathrm{C}$

D) Percentage of rings isolated from wild-type, myo2-S2 and cdc3-124 myo2-S2 cells that either contracted normally or presented slow/no contraction imaged at $24^{\circ} \mathrm{C}$. 
Figure 2

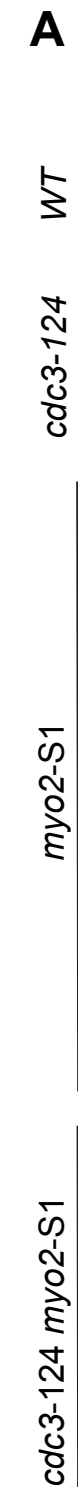

$\bar{c}$
ปิ
อิ

\section{$34^{\circ} \mathrm{C}$}
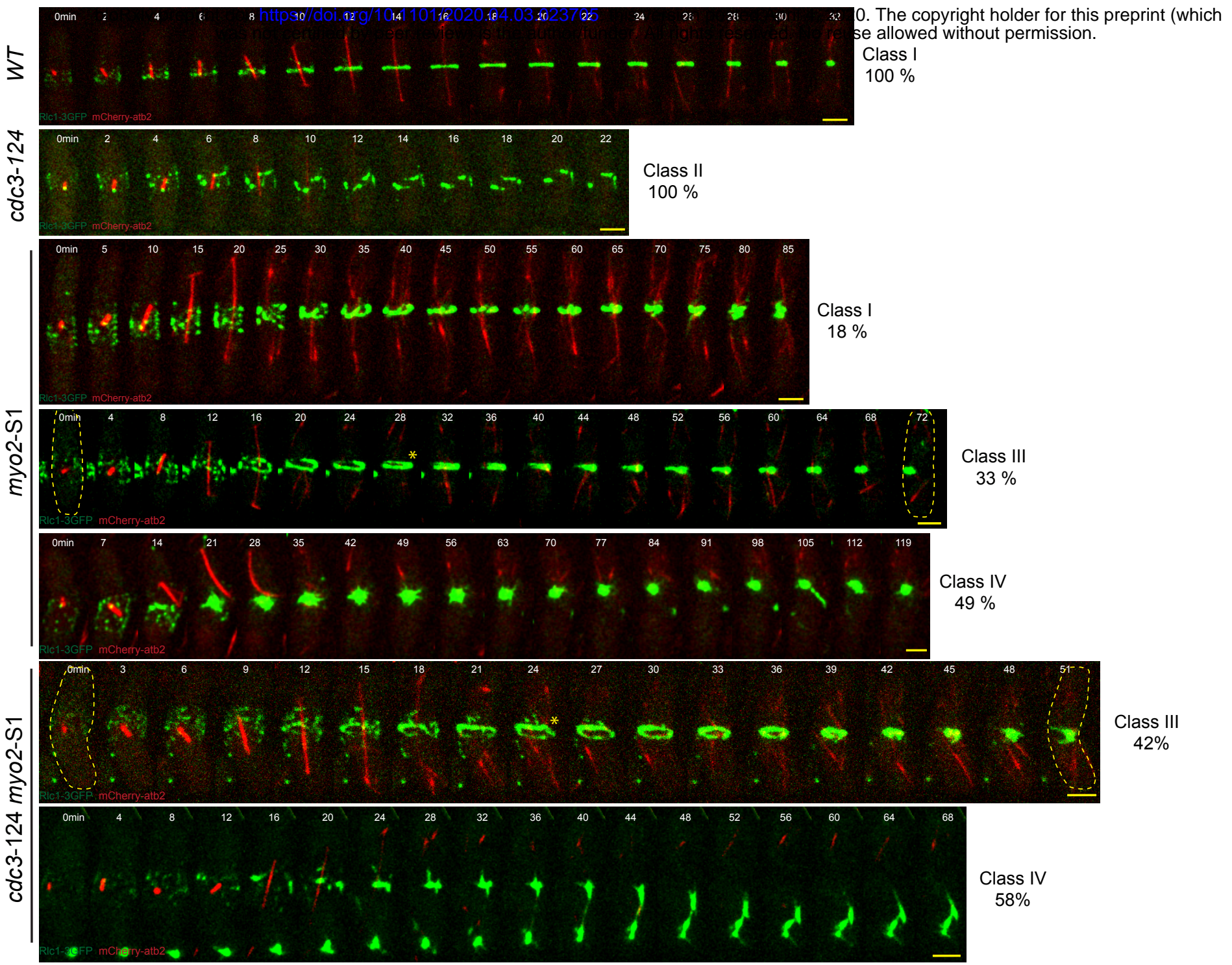

B

Actomyosin ring assembly time

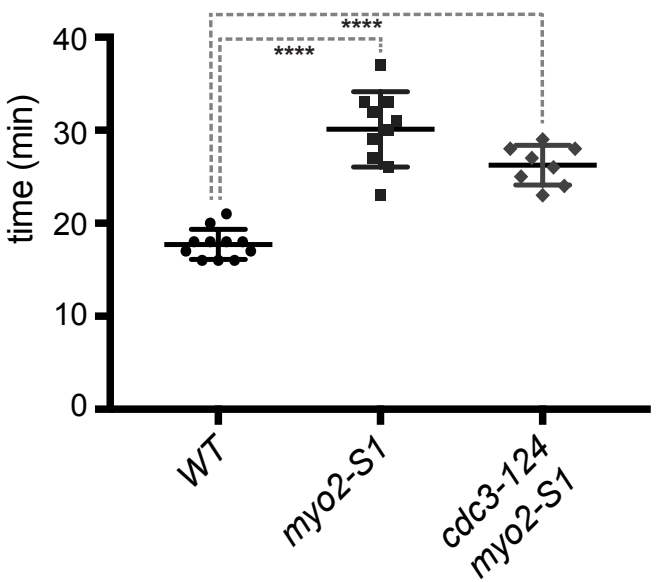

C

Actomyosin ring contraction time

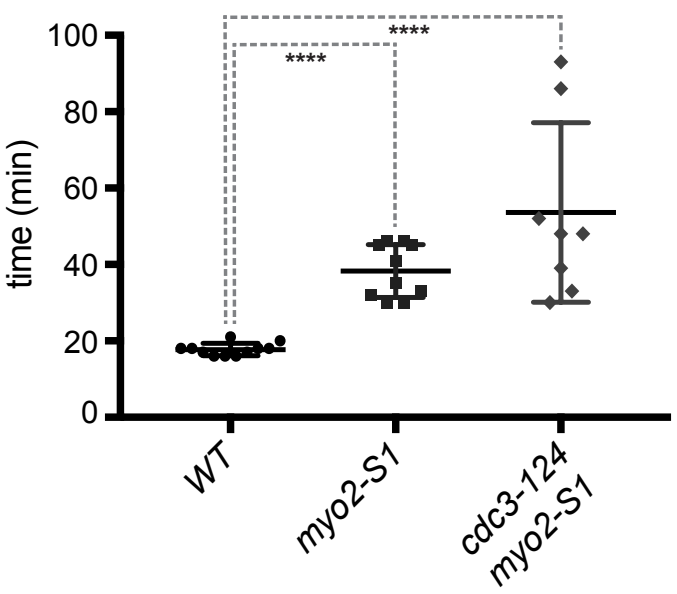


Figure 3

A

Myosin

HQ-Activation loop-HR

Actin

(barbed end)
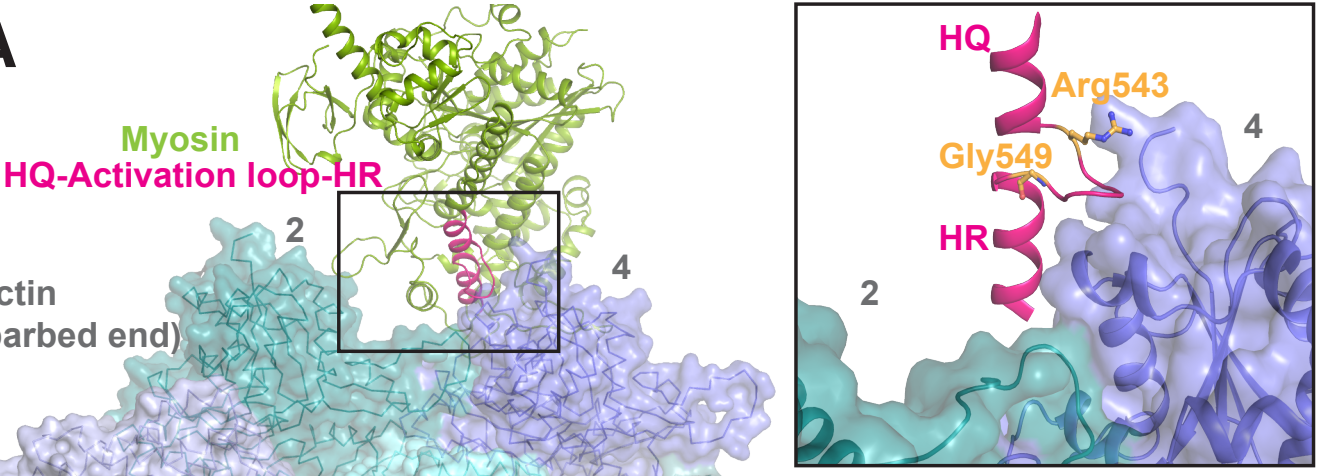

$\mathbf{B}$
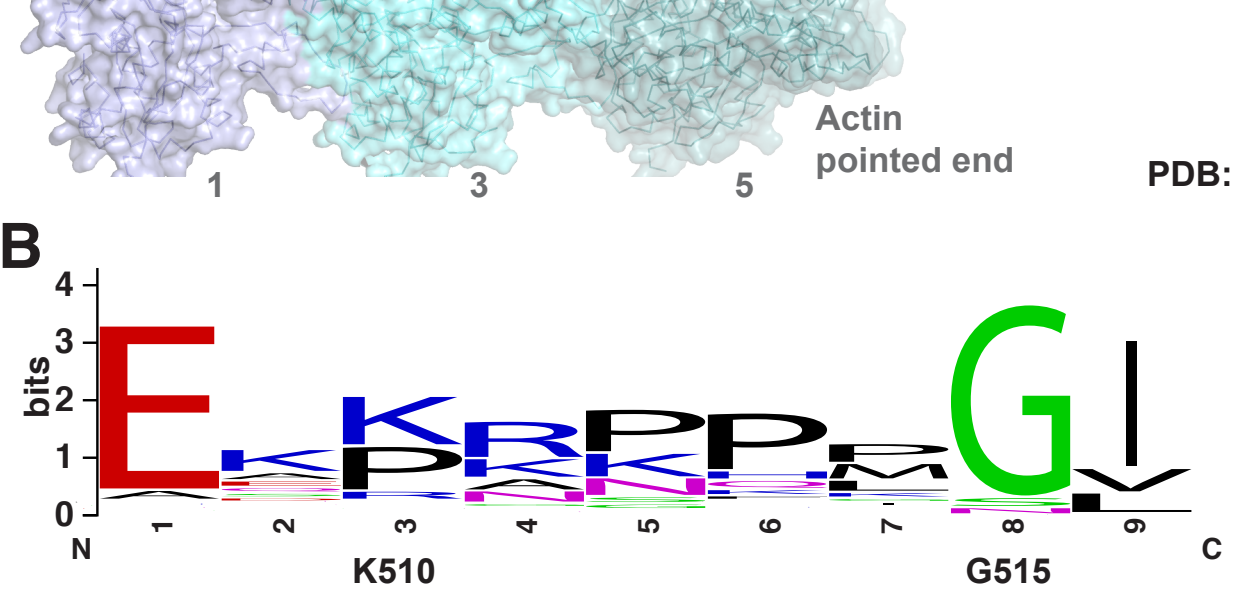

PDB: 5JLH 


\section{Figure 4}

A

36ioRxt plaspipt doi: https://doi.org/10.1101/2020.04.03.023705; this version posted April 4, 2020. The copyright holder for this preprint (which WT

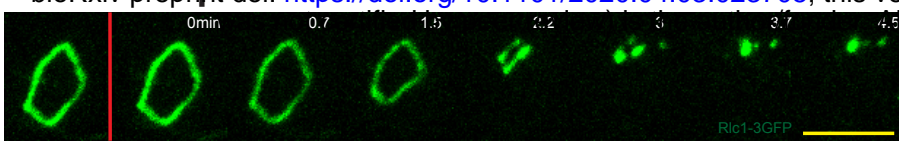

myo2-S1

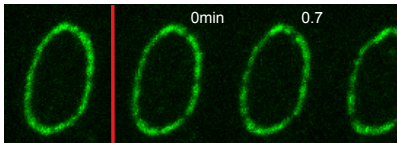

cdc3-124

myo2-S1

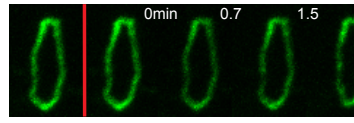

B

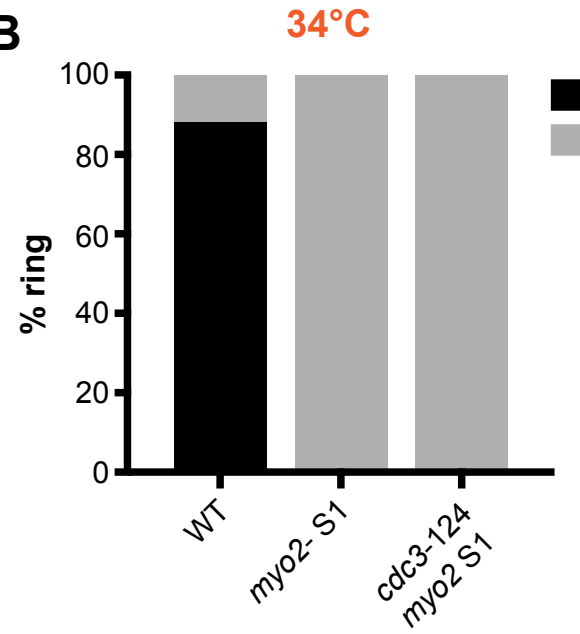

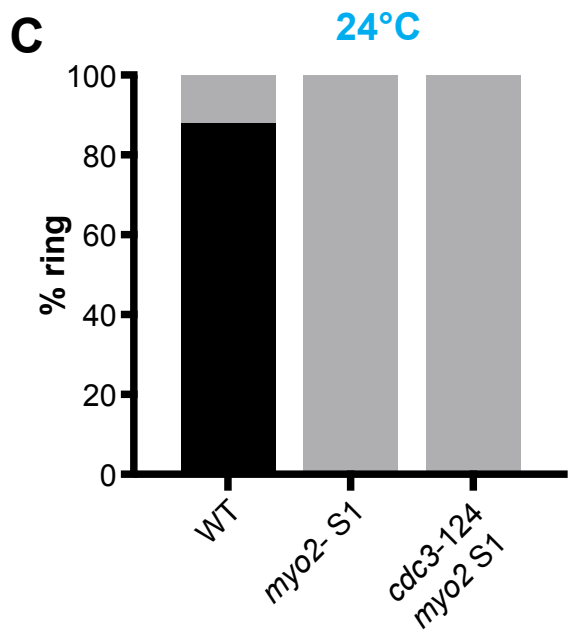

Normal contraction

Slow or no contraction
Slow or no contraction 
Figure 5
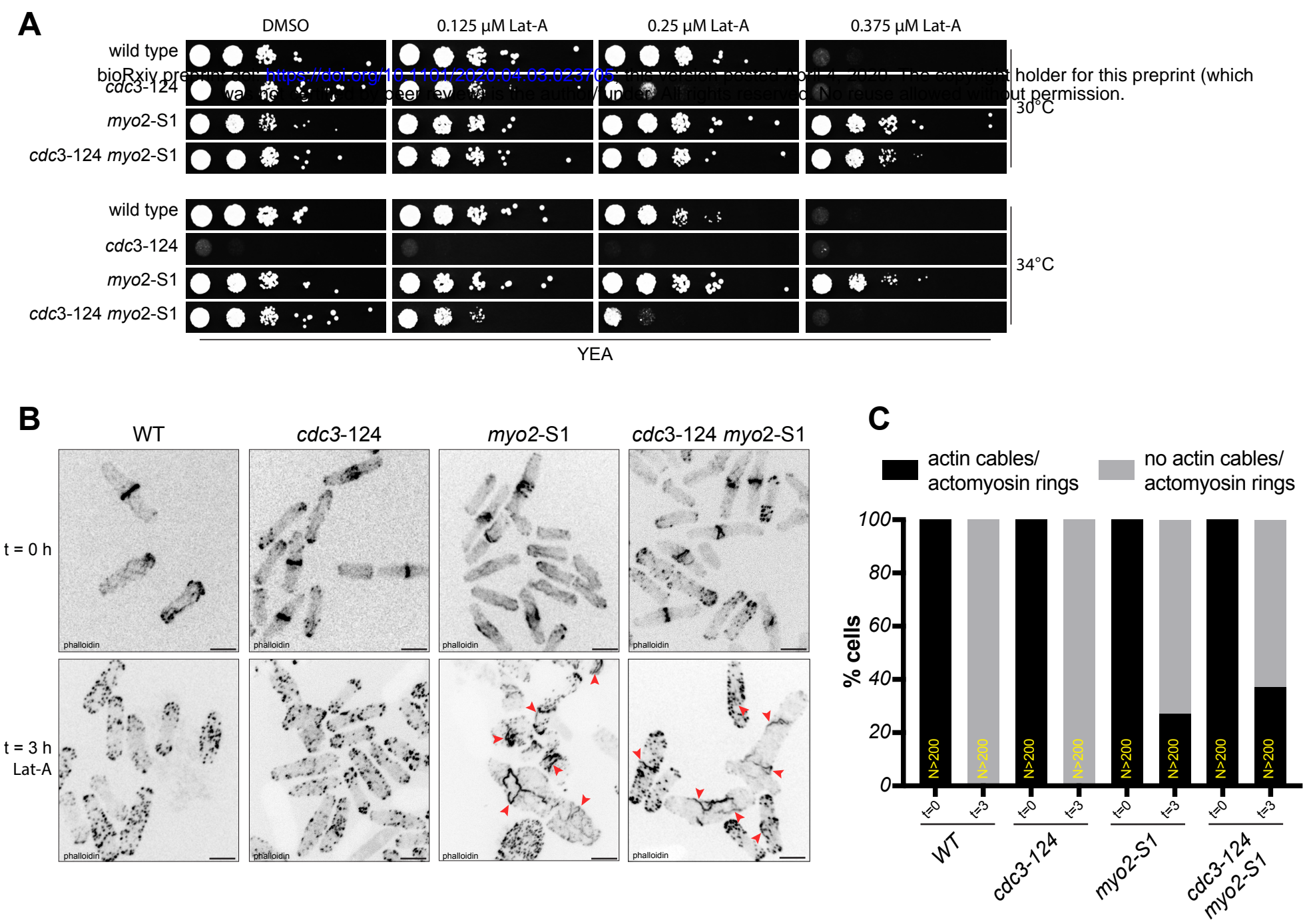